\title{
Corrigendum
}

\section{Corrigendum to "Integrated Wearable System for Monitoring Heart Rate and Step during Physical Activity"}

\author{
Eka Adi Prasetyo Joko Prawiro, ${ }^{1}$ Chun-I Yeh, ${ }^{1}$ Nai-Kuan Chou, ${ }^{2}$ \\ Ming-Wei Lee, ${ }^{1}$ and Yuan-Hsiang Lin ${ }^{1}$ \\ ${ }^{1}$ Department of Electronic and Computer Engineering, National Taiwan University of Science and Technology, Taipei 10607, Taiwan \\ ${ }^{2}$ Department of Surgery, National Taiwan University Hospital, Taipei 10048, Taiwan
}

Correspondence should be addressed to Yuan-Hsiang Lin; linyh@mail.ntust.edu.tw

Received 21 May 2017; Accepted 11 June 2017; Published 13 August 2017

Copyright (C) 2017 Eka Adi Prasetyo Joko Prawiro et al. This is an open access article distributed under the Creative Commons Attribution License, which permits unrestricted use, distribution, and reproduction in any medium, provided the original work is properly cited.

In the article titled "Integrated Wearable System for Monitoring Heart Rate and Step during Physical Activity" [1], the text appearing in the last paragraph of Section 2.5 should be corrected as follows: "A maximum value is set when the first fall signal is detected, and a minimum value is set when current input is higher than previous one." The magnitude and unit of $x$-axis data $(X)$ from Figure 8(a) should be corrected, as well as the magnitude and unit of integration process data $\left(X_{\text {int }}\right)$ and description of Figure $8(\mathrm{~b})$. For Table 1 , the header text and value in the first column should be corrected, as well as the header text in the second column.

Figure 8 and Table 1 are corrected as follows.

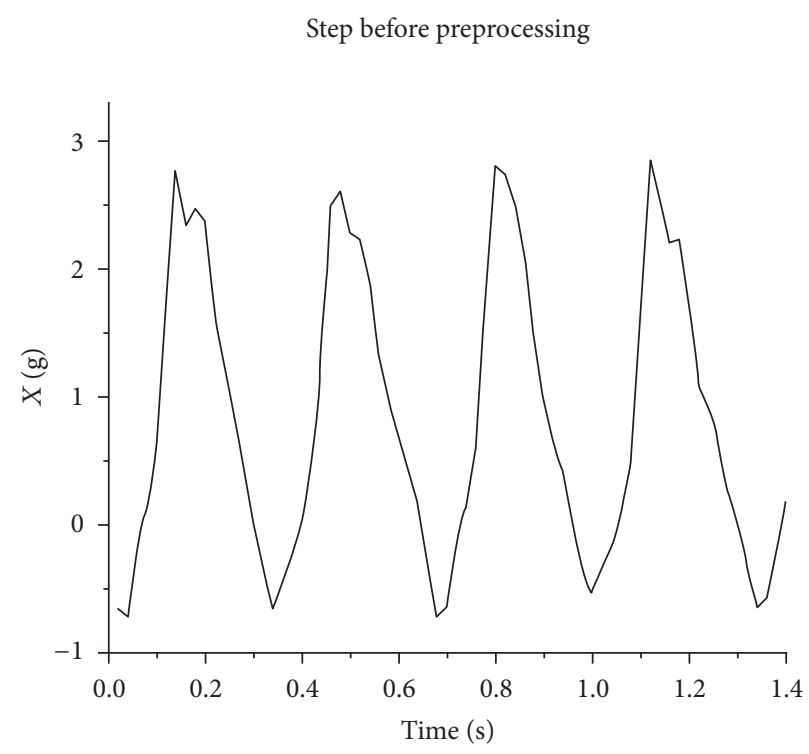

(a)

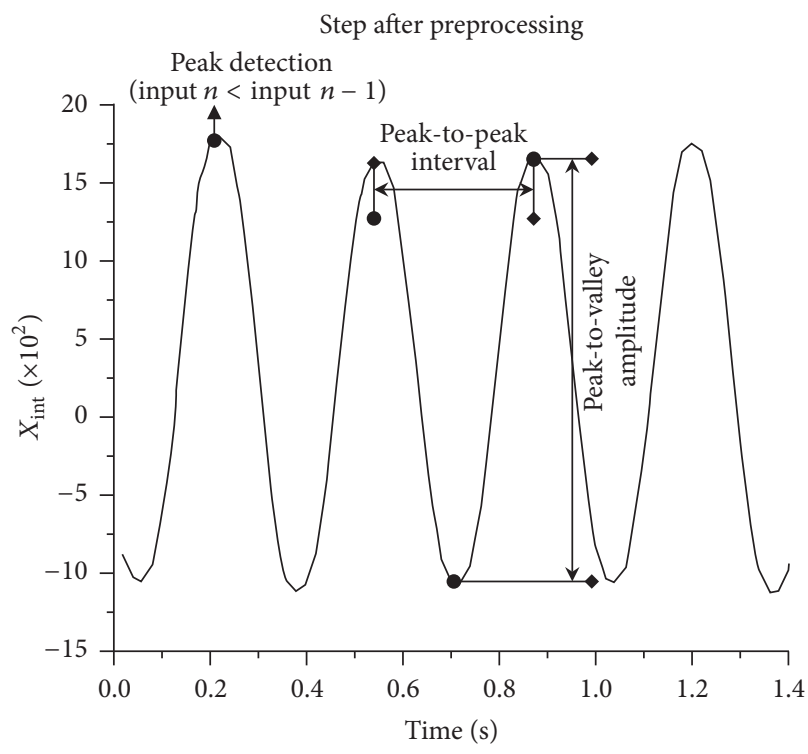

(b)

FIGURE 8: Step signal: (a) before preprocessing and (b) peak detection. 
TABLE 1: Relationship between peak-to-valley amplitude and adaptive threshold.

\begin{tabular}{lccc}
\hline Peak-to-valley amplitude of $X(\mathrm{~g})$ & Peak-to-valley amplitude of $X_{\text {int }}$ & Adapt. Thr. (number of samples (second)) & Walking status \\
\hline$<0.18$ & $<50$ & & Resting \\
$0.18 \sim 0.36$ & $50 \sim 200$ & $22(0.44)$ & Walking \\
$0.36 \sim 0.48$ & $200 \sim 300$ & $17(0.34)$ \\
$0.48 \sim 0.72$ & $300 \sim 500$ & $15(0.30)$ \\
$0.72 \sim 0.90$ & $500 \sim 650$ & $13(0.26)$ \\
$0.90 \sim 1.14$ & $650 \sim 850$ & $11(0.22)$ \\
$1.14 \sim 1.44$ & $850 \sim 1100$ & $10(0.20)$ & \\
$1.44 \sim 2.04$ & $1100 \sim 1600$ & $9(0.18)$ & Running \\
$>2.04$ & $>1600$ &
\end{tabular}

\section{References}

[1] E. A. P. J. Prawiro, C.-I. Yeh, N.-K. Chou, M.-W. Lee, and Y.-H. Lin, "Integrated wearable system for monitoring heart rate and step during physical activity," Mobile Information Systems, vol. 2016, Article ID 6850168, 10 pages, 2016. 

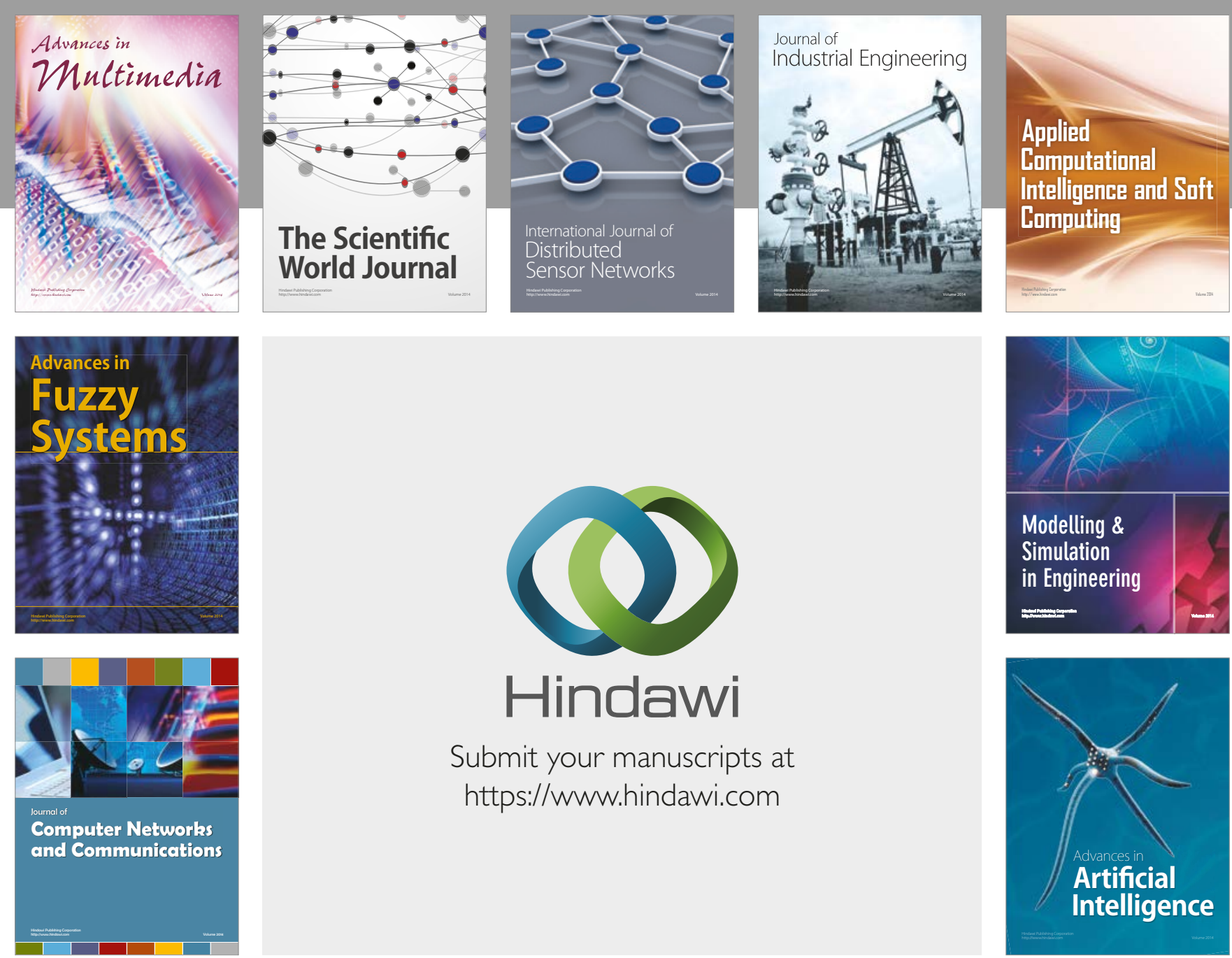

\section{Hindawi}

Submit your manuscripts at

https://www.hindawi.com
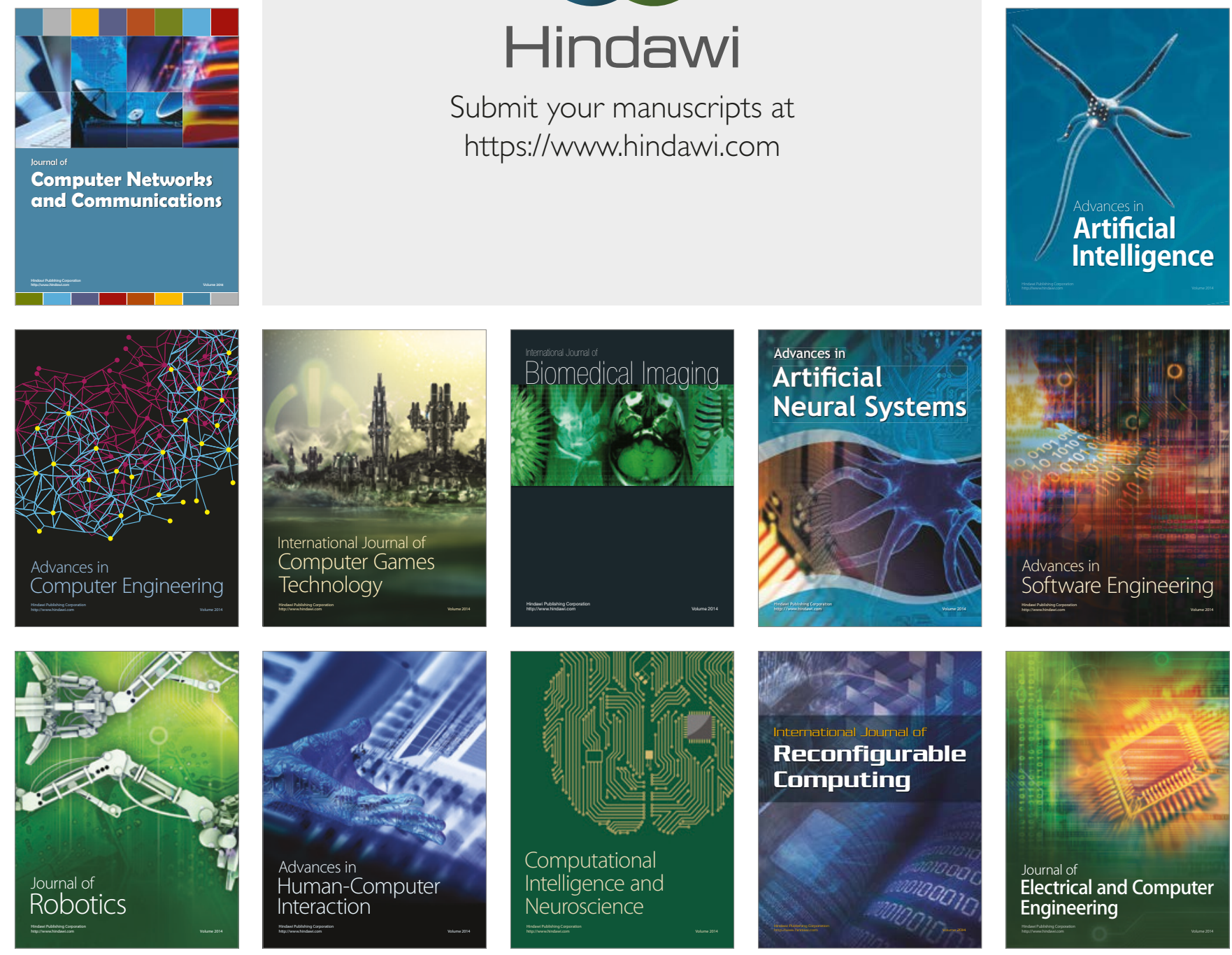